\title{
Randomized controlled trial of geriatric consultation versus standard care in older adults with hematologic malignancies
}

\author{
Clark DuMontier, ${ }^{1,2,3}$ Hajime Uno, ${ }^{3,4}$ Tammy Hshieh, ${ }^{1,3,5}$ Guohai Zhou, ${ }^{1,3}$ Richard \\ Chen, ${ }^{5}$ Emily S. Magnavita, ${ }^{5}$ Lee Mozessohn, ${ }^{6}$ Houman Javedan, ${ }^{1,3}$ Richard M. \\ Stone ${ }^{3,5}$ Robert J. Soiffer, ${ }^{3,5}$ Jane A. Driver ${ }^{1,2,3 \#}$ and Gregory A. Abe ${ }^{3,54}$
}

${ }^{1}$ Division of Aging, Brigham and Women's Hospital, Boston, MA, USA; ${ }^{2} N e w$ England Geriatric Research Education and Clinical Center, VA Boston Healthcare System, Boston, MA, USA; ${ }^{3}$ Harvard Medical School, Boston, MA, USA; ${ }^{4}$ Department of Data Sciences, Dana-Farber Cancer Institute, Boston, MA, USA; ${ }^{5}$ Department of Medical Oncology, DanaFarber Cancer Institute, Boston, MA, USA and ${ }^{6}$ Sunnybrook Odette Cancer Centre, Toronto, Ontario, Canada

"JAD and GAA contributed equally as co-senior authors.

\section{ABSTRACT}

W e conducted a randomized controlled trial in older adults with hematologic malignancies to determine the impact of geriatrician consultation embedded in our oncology clinic alongside standard care. From February 2015 to May 2018, transplant-ineligible patients aged $\geq 75$ years who presented for initial consultation for lymphoma, leukemia, or multiple myeloma at Dana-Farber Cancer Institute (Boston, MA, USA) were eligible. Pre-frail and frail patients, classified based on phenotypic and deficit-accumulation approaches, were randomized to receive either standard oncologic care with or without consultation with a geriatrician. The primary outcome was 1-year overall survival. Secondary outcomes included unplanned care utilization within 6 months of follow-up and documented end-of-life (EOL) goals-of-care discussions. Clinicians were surveyed as to their impressions of geriatric consultation. One hundred sixty patients were randomized to either geriatric consultation plus standard care $(n=60)$ or standard care alone $(n=100)$. The median age of the patients was 80.4 years (standard deviation $=4.2$ ). Of those randomized to geriatric consultation, $48(80 \%)$ completed at least one visit with a geriatrician. Consultation did not improve survival at 1 year compared to standard care (difference: $2.9 \%, 95 \%$ confidence interval: $-9.5 \%$ to $15.2 \%, P=0.65)$, and did not significantly reduce the incidence of emergency department visits, hospital admissions, or days in hospital. Consultation did improve the odds of having EOL goals-of-care discussions (odds ratio $=3.12,95 \%$ confidence interval: 1.03 to 9.41 ) and was valued by surveyed hematologic-oncology clinicians, with $62.9 \%-88.2 \%$ of them rating consultation as useful in the management of several geriatric domains.

\section{Introduction}

Older adults constitute the majority of patients with hematologic malignancies, as the median ages at diagnosis of non-Hodgkin lymphoma, leukemia, and multiple myeloma are 67,67 , and 69 years, respectively. ${ }^{1-3}$ Compared with younger patients, older patients with blood cancers often have age-related vulnerabilities that complicate their care. ${ }^{4}$ Cognitive impairment, functional dependency, and frailty are prevalent and associated with worse outcomes such as increased treatment toxicity, unplanned hospitalizations, and higher mortality. ${ }^{5.7}$ To manage this complexity, cancer organizations such as the American Society of Clinical Oncology (ASCO) recommend that all older adults with cancer treated with chemotherapy undergo a geriatric assessment (GA): a multidisciplinary evaluation of domains necessary for older adult health and well-being. ${ }^{8}$ 
There is strong evidence from observational studies that, in comparison with standard oncologic assessment, GA better identifies age-related vulnerabilities, guides the care of these vulnerabilities, influences treatment decisions, and predicts outcomes in older patients with cancer. ${ }^{9-14}$ Moreover, recent randomized controlled trials suggest that GA-guided interventions may reduce treatment toxicity, improve quality of life, and improve communication with patients and caregivers. ${ }^{15-17}$ Unfortunately, these trials include mainly older patients with solid tumors. To our knowledge, no similar trials in patients with blood cancers have been reported. We thus leveraged the embedded geriatrics resources available in our outpatient blood cancer clinic to determine the impact of consultation with a geriatrician alongside standard oncologic care for patients aged 75 and older with hematologic malignancies.

\section{Methods}

\section{Patients and study design}

This randomized controlled trial enrolled patients from February 2015 to May 2018 (ClinicalTrials.gov identifier NCT02359838) (Online Supplementary File S1). Eligible patients included all patients aged 75 years and older who presented to Dana-Farber Cancer Institute (Boston, MA, USA) for initial consultation seeking management for newly diagnosed or previously diagnosed and treated lymphoma, leukemia, or multiple myeloma. Patients were ineligible if they were referred for consultation for stem cell transplantation or did not plan to continue their care at our institution. Eligible patients who consented to participate in the study underwent an in-person screening GA administered by a research assistant on the same day as their initial hematologic oncology consultation, as described previously. ${ }^{5}$ From this assessment, frailty status was derived using both the phenotypic and deficit-accumulation approaches - two of the most widely-studied approaches in aging research (see the protocol in Online Supplementary File $S 1$ for further details regarding these approaches and their cut-off values that classified severity of frailty). ${ }^{18,19}$ In brief, the frailty phenotype uses five criteria to define a syndrome (slow gait, weakness [grip strength], self-reported exhaustion, low physical activity, and weight loss; average time to complete, 5-10 minutes). The deficit-accumulation method counts numerous aging-related health deficits across multiple domains from a GA to define frailty as the proportion of deficits present in an individual out of the total number of possible deficits measured (average time to complete, 15-20 minutes). We did not use a disease-specific frailty score such as the International Myeloma Working Group score. Patients classified as pre-frail or frail by either approach were randomized to either standard oncologic care as they would normally receive at Dana-Farber or standard care plus embedded consultation provided by a geriatrician. All oncologists were blinded to the initial geriatric screening and frailty classification, precluding an influence on initial treatment recommendations. Oncologists of patients in the intervention group may have become aware of patients' frailty status later in the study after the patients had been assessed by the geriatrician.

Randomization was stratified by disease type to minimize potential imbalances in blood cancers and treatments. Randomization was first conducted on a 1:1 ratio but was switched to a 2:1 ratio (standard care: standard care plus geriatric consultation) to increase enrollment, which was initially limited due to difficulties in scheduling patients assigned to the intervention arm to one of the twice-weekly geriatric clinic sessions. The study was powered to detect a difference in 1-year overall survival (the primary outcome) of $25 \%$ between study arms, hypothesizing that the geriatric consultation arm would demonstrate this percent improvement in survival. This effect size was estimated based on prior observational data regarding survival rates in similar patients treated at Dana-Farber and the survival benefit associated with integrated palliative care in patients with lung cancer. ${ }^{20}$ With 2:1 randomization, the sample size needed to detect the estimated effect size was calculated to be $160-107$ in the standard care arm and 53 in the arm with geriatric consultation - assuming $80 \%$ power and a one-sided type I error rate of $5 \%$. This sample size was recalculated from 152 (76 per group), which was originally calculated for 1:1 randomization. The study was approved by the Dana-Farber/Harvard Cancer Center Office for the Protection of Human Research Subjects.

\section{Geriatric consultation intervention}

Patients who were assigned to the intervention received embedded geriatric consultation with a licensed geriatrician in addition to their standard oncologic care managed by their hematologic oncologist. The embedded geriatrics clinic is located within DanaFarber on the same floors as the hematologic malignancies clinics. Of note, embedded geriatric consultation had been available for patients referred from the leukemia clinic (without prior GA screening and randomization) for 2 years preceding the start of the trial.

After assignment, patients from leukemia, lymphoma, and myeloma clinics were scheduled with a geriatrician either on the same day as their follow-up oncology consultation or at a different time in accordance with the patient's schedule and appointment availability; we intended for the patients to be seen as early as possible but did not require a specific time period for the first visit. Consistent with other trial designs evaluating the effectiveness of integrated subspecialty care,$^{20}$ the geriatrician provided further management and interventions individualized to the patient based on clinical judgment and best-available evidence; no pre-specified interventions were required. If indicated, geriatricians communicated with the patient's primary care provider and utilized referral systems (e.g., physical therapy, psychiatry) already established at Dana-Farber. Follow-up appointments were encouraged, but not required.

In keeping with routine care provided by geriatricians, the geriatrician conducted a GA for every patient encountered. To characterize the interventions recommended by the geriatrician, a content analysis of the geriatricians' notes was conducted. ${ }^{21}$ For each patient we classified whether the geriatrician recommended an intervention targeting one or more domains described in ASCO's Guideline for Geriatric Oncology: (i) function and falls; (ii) comorbidity and polypharmacy; (iii) cognition; (iv) depression/mood; and (v) nutrition. ${ }^{8}$ Recommended interventions could include counseling, recommendations for non-pharmacological interventions, pharmacological interventions, and referrals to other specialties or allied healthcare. For each patient, all geriatricians' notes through the 1-year follow-up period were reviewed, and new interventions were only counted once.

\section{Outcome measures}

The primary outcome of this study was 1-year overall survival from the time of initial hematologic oncology consultation. Vital status was confirmed by a combination of chart review and calls to patients' primary care providers.

Secondary outcomes were assessed via chart review and included the number of emergency department visits, the number of unplanned hospital admissions, and the number of days spent in the hospital ${ }^{22}$ within 6 months after patients' initial consultations at Dana-Farber. Having any end-of-life (EOL) goals-of-care discus- 
sions documented in the medical record during the 1-year followup period was also measured via chart review. EOL goals-of-care discussion was defined as a discussion regarding EOL preferences by any treating clinician including resuscitation/code status, hospice, and/or preferred location for dying. ${ }^{23}$ Finally, after completion of enrollment, a survey was administered to 65 Dana-Farber hematologic oncologists, physician assistants, and nurse practitioners who cared for patients in the geriatric consultation arm. This survey sought to gather clinicians' opinions regarding the usefulness of consultation (on a Likert scale where 1 = "least" useful and $5=$ "most" useful) in addressing geriatric domains of care and areas of management for specific age-related issues (see survey instrument, Online Supplementary Figure S2).

\section{Statistical analysis}

For the primary analysis, the impact of geriatric consultation on 1-year overall survival was assessed using Kaplan-Meier analysis comparing the 1-year survival rate between patients receiving geriatric consultation plus standard oncologic care and patients receiving standard oncologic care alone. Differences in 1-year survival rate and a corresponding $95 \%$ confidence interval (95\% CI) were calculated to summarize the effect of geriatric consultation on 1-year overall survival. ${ }^{24,25}$ Multivariable Cox regression and weighted logistic regression models ${ }^{26}$ were also used to estimate the treatment effect, adjusting for any potential remaining imbalances after randomization related to age, sex, disease aggressiveness (defined according to previous methods ${ }^{5-7}$ ), and frailty (prefrail versus frail). Aggressive diseases included diffuse large B-cell lymphoma, mantle cell lymphoma, multiple myeloma, acute myeloid leukemia, and indolent diseases included marginal zone lymphoma, follicular lymphoma, chronic lymphocytic leukemia, myelodysplastic syndrome, myeloproliferative neoplasm/myeloproliferative disease, and hairy-cell leukemia.

For secondary analyses, the effects of geriatric consultation on emergency department visits, hospitalizations, and number of days in the hospital were assessed using separate negative binomial regression models, each adjusting for age, sex, disease aggressiveness, and frailty. The impact of geriatric consultation on the likelihood of having documented EOL goals-of-care discussions during the follow-up period was assessed using multivariable logistic regression, adjusting for the aforementioned covariates. Exploratory analyses investigated any association between number of geriatrician visits and mortality, as well as a subgroup analysis determining any difference in effect by frailty severity. All primary and secondary analyses were performed as intention-totreat analyses, followed by per-protocol analyses that excluded patients who, although assigned to the intervention, ended up not completing their geriatric visit. SAS (version 9.4, SAS Institute, Cary, NC, USA) and R (version 4.0,0, https://www.R-project.org, R foundation for Statistical Computing, Vienna, Austria) statistical software were used for all analyses.

\section{Results}

\section{Patients' characteristics}

Between February 2015 and May 2018, 270 eligible patients with planned follow-up at Dana-Farber were approached for enrollment (Figure 1). Of these, 232 agreed to participate and underwent the screening GA, after which 72 patients were classified as robust and thus excluded from the trial. One hundred sixty pre-frail/frail patients were randomly assigned to receive geriatric consultation plus standard oncologic care $(n=60)$ or standard care alone $(n=100)$. One patient in the standard care arm was lost to follow-up because the patient never returned to Dana-Farber after initial consult and vital status could not be confirmed. This patient was assumed to be alive at the end of the study period and was included in the analyses. In the intervention arm, three patients died before receiving their consultation, three cancelled the consultation, and six did not return to Dana Farber (i.e., they continued their care at their local practice). The two study arms were overall balanced in terms of baseline characteristics (Table 1), with high rates of functional impairment (35.6\% with dependency in instrumental activities of daily living [IADL]), cognitive impairment $(39.5 \%$ with impairment in executive function), and mobility impairment $(60.6 \%$ with gait speed $<0.8$ meters/second). Online Supplementary Table S1 lists the latest active treatment regimens within 3 months of initial consultation.

\section{Uptake of the embedded geriatric consultation}

Of those randomized to geriatric consultation, 48 $(80 \%)$ completed at least one visit with a geriatrician ( $95 \%$ CI: $68 \%$ to $88 \%$ ). Of those 12 assigned to receive geriatric consultation who did not complete it, three died, three cancelled the consultation (although continued their cancer care at Dana-Farber), and six ended up not returning to Dana-Farber for further care. Among the 48 who completed at least one consultation, 26 completed one or more additional visits with a geriatrician (range of total visits per patient, 1-12). Patients enrolled toward the end of the study period tended to have more total visits than patients enrolled toward the beginning (Online Supplementary Figure S2).

\section{Geriatric consultation and 1-year overall survival}

After being randomized to the geriatric consultation arm, time to the initial visit with a geriatrician varied across patients with a median of 36 days (range, 0-224 days; interquartile range, 76 days). The median follow-up extended beyond our outcome of 1-year survival. Among the 48 patients who were seen by the geriatrician in the consultation arm, the median number of interventions recommended for each patient was two, with a range of zero to four interventions. The most common interventions fell within the comorbidity/polypharmacy domain (39 [81.3\%] patients receiving one or more interventions); followed by nutrition (26 [54.2\%]); function/falls (23 [47.9\%]); cognition (15 $[31.3 \%]) ;$ and depression/mood $(8[16.7 \%])$. Ninetyseven of these interventions were carried out by the geriatrician through counseling, non-pharmacological recommendations, or pharmacological prescriptions. Fourteen of these interventions were referrals or coordination with other disciplines, including physical therapists, social workers, and nutritionists. No control patients crossed over to the consultation arm in the 1-year follow-up period (i.e., no control patient received an embedded geriatric consultation).

A cumulative total of 32 patients died in the year following their initial consultation, $11(18.3 \%)$ in the geriatric consultation arm versus $21(21.0 \%)$ in the standard care arm. Overall survival at 1 year was not significantly higher in patients receiving geriatric consultation $(81.7 \%, 95 \% \mathrm{CI}$ : $71.0 \%$ to $90.2 \%$ ) in comparison with patients receiving standard care $(78.8 \%, 95 \%$ CI: $69.7 \%$ to $85.7 \%$; difference: $2.9 \%, 95 \%$ CI: $-9.5 \%$ to $15.2 \%, P=0.65$ ) (Figure $2 \mathrm{~A}$ ). Results were similar in the per-protocol analysis (Figure 2B), as were results after adjustment for covariates in the multi- 
variable analyses (Table 2, Online Supplementary Tables S2 and S3). Moreover, there was no significant association between the number of visits with a geriatrician and mortality (hazard ratio $=0.78,95 \%$ CI: 0.43 to 1.39 ), and there was no difference in the effect of consultation on mortality among frail versus pre-frail patients (test for interaction $P=0.41$ ). Frail patients experienced higher mortality, independently of intervention or other covariates (Online Supplementary Table S2).

\section{Geriatric consultation and acute care utilization}

Thirty-six of 160 patients $(22.5 \%)$ experienced one or more unplanned hospitalizations during the first 6 months of follow-up, and the same number made one or more emergency department visits. In comparison with patients who received standard oncologic care, patients who received geriatric consultation did not have a significantly lower incidence of emergency department visits (incident rate ratio $[\mathrm{RR}]=0.89,95 \% \mathrm{CI}: 0.33$ to 2.42 ), hospitalizations (IRR $=0.91,95 \%$ CI: 0.30 to 2.71 ), or days spent in hospital (IRR $=1.05,95 \%$ CI: 0.29 to 3.79 ), adjusting for covariates (Table 2). Per-protocol analyses yielded similar results.

\section{Geriatric consultation and end-of-life goals-of-care dis- cussions}

Seventeen of 160 patients (10.6\%) received one or more EOL goals-of-care discussions during follow-up. In comparison with patients who received standard oncologic care, patients who received geriatric consultation had an over three-fold higher odds of a documented goals-of-care discussion (odds ratio $=3.12,95 \%$ CI: 1.03 to 9.41). Per-protocol analyses yielded similar results (odds ratio $=3.58,95 \%$ CI: 1.13 to 11.35 ). Three patients in each arm received a palliative care consultation.

\section{Hematologic oncologists' and other clinicians' perceived value of geriatric consultation}

Thirty-five of $65(53.8 \%)$ hematologic oncologists, nurse practitioners, and physician assistants whose patients had

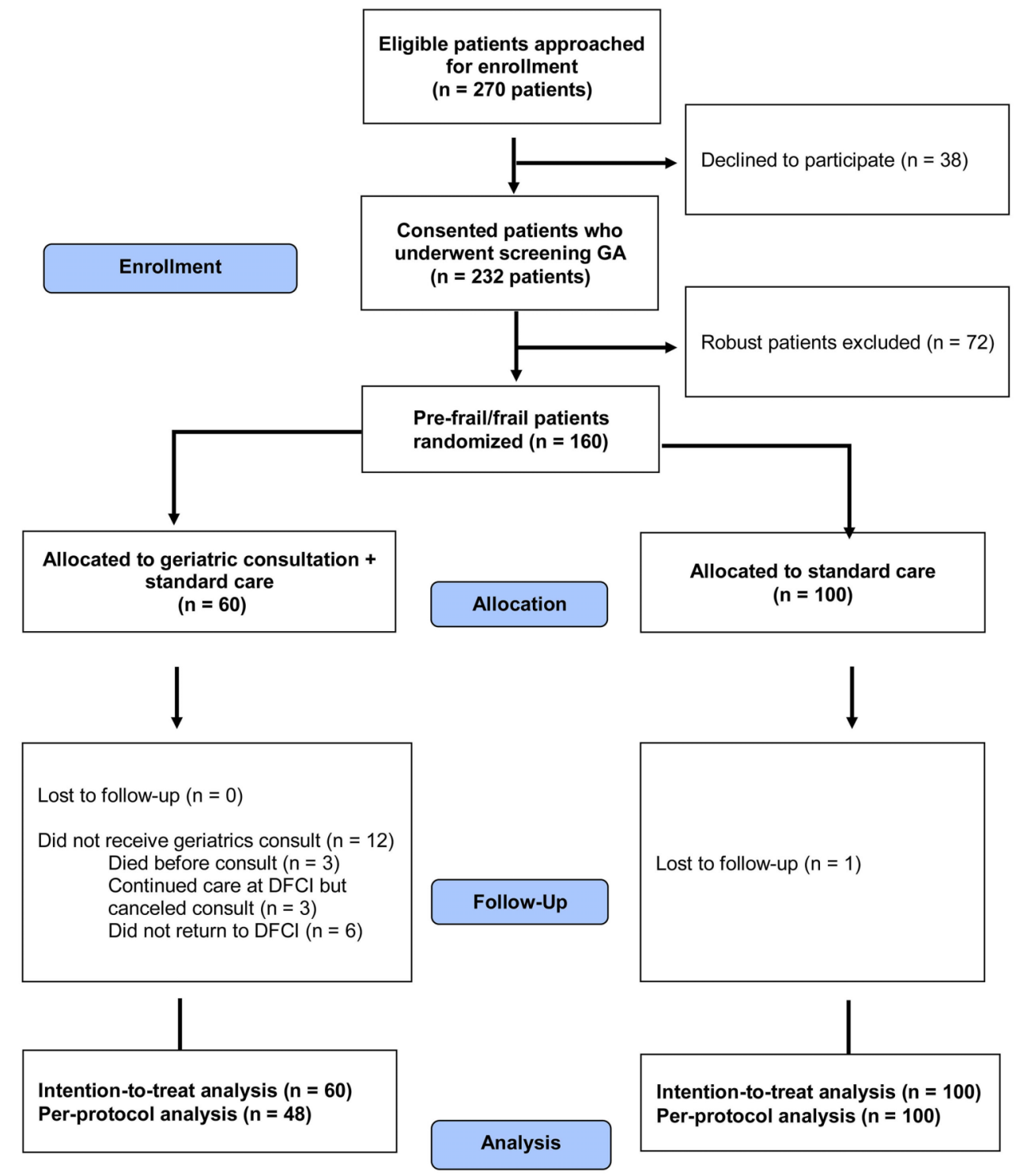

Figure 1. CONSORT flow diagram of trial enrollment and analysis. DFCl: Dana-Farber Cancer Institute; GA: geriatric assessment. 
received geriatric consultation responded to the survey evaluating the perceived value of geriatric recommendations. The majority found embedded geriatric consultation to be valuable in managing several age-related domains of care (Table 3). Domains of care in which consultation was found to be most valued included evaluation of cognition, connecting patients to resources, diagnosing frailty, and managing non-oncologic comorbidities. Specific areas of management found to be most useful included optimizing functional status, treating falls, and treatment of depression and other mood disorders.

\section{Discussion}

We found that in pre-frail and frail older patients with hematologic malignancies, embedded geriatric consultation did not improve 1-year overall survival or acute care utilization. However, consultation significantly increased the likelihood of having EOL goals-of-care discussions. Moreover, hematologic oncology clinicians highly valued the services provided by geriatrics in the care of their older patients. Our trial addresses a critical gap regarding the effectiveness of geriatric-driven interventions in older patients with hematologic malignancies, complementing emerging evidence from other studies predominantly including older adults with solid tumors. ${ }^{15-17,27-31}$

Few prior studies have investigated GA-driven interventions for patients with blood cancers. Artz and colleagues recently reported that in patients with a median age of 67 years undergoing hematopoietic stem cell transplantation for blood cancers, GA-driven interventions implemented by a multidisciplinary geriatrics team improved 1-year overall survival in comparison with conducting a GA alone without a multidisciplinary team to manage any detected vulnerabilities. ${ }^{32}$ While provocative, a limitation of this nonrandomized study was its use of a historical control group for comparison. In our randomized trial of transplant-ineligible patients aged $\geq 75$ years undergoing geriatric consultation versus standard oncologic care, we did not find evidence of an effect on 1-year overall survival, even in the frail subgroup.

Care for frail older adults is often complex and fragmented. ${ }^{33} \mathrm{~A}$ significant strength of our consultative model is that our geriatricians - trained specialists in frailty and complex care - were embedded in our center, caring for patients alongside their hematologic oncologists in the same clinic. On the other hand, certain aspects of our model may have limited its effectiveness in both reducing mortality and acute care utilization. First, although $80 \%$ of patients assigned to receive consultation ended up completing the consultation, our challenges in enrolling and assigning patients to the consultation arm reflected the limited capacity of geriatricians in our clinic. Relatedly, we found the time to have the initial visit with a geriatrician varied across patients, with a median of 36 days. Delays were largely a function of the patients' busy schedules and the fact that our geriatrics clinics only occurred twice per week. Second, the geriatricians worked within the established referral structures existing at Dana-Farber rather than with a dedicated multidisciplinary team (e.g., including pharmacists, social workers, and allied health specialties), which may have limited the breadth and timeliness of any geriatricsrecommended interventions. ${ }^{34,35}$ Lastly, the utilization of our geriatric consultation service evolved over the study period, with more clinicians requesting longitudinal co-management, rather than a single consultation, later in the study. We implicitly intended more longitudinal management for all patients assigned to the geriatrics intervention but found that just over half received additional follow-up visits.

The effectiveness of longitudinal geriatric co-management models delivered earlier in follow-up, with or without multidisciplinary support, warrants further investigation in frail older adults with blood cancers. Preliminary findings from such a geriatrician-led model in older adults with mostly solid tumors are encouraging, showing - in contrast to our study - a significant reduction in emergency presentations and unplanned hospitalizations in comparison to usual care. ${ }^{36}$ Important distinctions between this model and ours are worth noting. Patients' initial visit with the geriatrician occurred upon enrollment in the study, which ensured earlier delivery of any GA-driven interventions. Additionally, although the intervention design, like ours, allowed for individualized management tailored by the geriatrician, standardized interventions were provided to all patients assigned to the geriatrics arm that included supportive care information and optimization of physical activity and nutrition. Lastly, more longitudinal co-management occurred than in our study's consultative model, with patients receiving reassessments at multiple points in their follow-up period. Although we did not find an association between number of visits and mortality, our study was underpowered to formally analyze this association. Moreover, the geriatrician may have elected to see sicker patients more often, confounding the association. Earlier delivery, more integration, and more longitudinal follow-up

Table 1. Baseline characteristics of the study population.

\begin{tabular}{|c|c|c|c|}
\hline Characteristic & $\underset{(n=160)}{A}$ & $\begin{array}{c}\text { Standard } \\
\text { oncologic care } \\
(n=100)\end{array}$ & $\begin{array}{c}\text { Geriatric } \\
\text { consultation + } \\
\text { standard care } \\
(n=60)\end{array}$ \\
\hline Age, mean (SD) & $80.4(4.2)$ & $80.3(3.9)$ & $80.5(4.7)$ \\
\hline Male, n. (\%) & $104(65.0)$ & $64(64.0)$ & 40 (66.7) \\
\hline $\begin{array}{l}\text { Disease type, n. (\%) } \\
\text { Lymphoid } \\
\text { Myeloid } \\
\text { Myeloma }\end{array}$ & $\begin{array}{l}50(31.3) \\
48(30.0) \\
62(38.8)\end{array}$ & $\begin{array}{l}36(36.0) \\
28(28.0) \\
36(36.0)\end{array}$ & $\begin{array}{l}14(23.3) \\
20(33.3) \\
26(43.3)\end{array}$ \\
\hline Aggressive disease, n. (\%) & $60(37.5)$ & $37(37.0)$ & $23(38.3)$ \\
\hline $\begin{array}{l}\text { Frailty, n. (\%) } \\
\text { Pre-frail } \\
\text { Frail }\end{array}$ & $\begin{array}{l}124(77.5) \\
36(22.5)\end{array}$ & $\begin{array}{l}75(75.0) \\
25(25.0)\end{array}$ & $\begin{array}{l}49(81.7) \\
11(18.3)\end{array}$ \\
\hline $\begin{array}{l}\text { Gait speed, } \mathrm{n} .<0.8 \mathrm{~m} / \mathrm{s}(\%) \\
\text { Declined/missing }\end{array}$ & $\begin{array}{c}97(60.6) \\
4(2.5)\end{array}$ & $\begin{array}{c}60(60.0) \\
3(3.0)\end{array}$ & $\begin{array}{l}37(61.7) \\
1(1.7)\end{array}$ \\
\hline Cognition, $n$. with impairmen & $(\%)$ & & \\
\hline $\begin{array}{l}\text { Delayed recall } \\
\text { Declined/missing } \\
\text { Executive function } \\
\text { Declined/missing }\end{array}$ & $\begin{array}{c}25(16.0) \\
4(2.5) \\
60(39.5) \\
8(5.0)\end{array}$ & $\begin{array}{c}16(16.7) \\
4(4.0) \\
38(40.9) \\
7(7.0)\end{array}$ & $\begin{array}{c}9(15.0) \\
0(0.0) \\
22(37.3) \\
1(1.7)\end{array}$ \\
\hline $\begin{array}{l}\text { Function, n. with impairment } \\
\text { ADL } \\
\text { IADL }\end{array}$ & $\begin{array}{l}\%) \\
27(16.9) \\
57(35.6)\end{array}$ & $\begin{array}{l}18(18.0) \\
34(34.0)\end{array}$ & $\begin{array}{c}9(15.0) \\
23(38.3)\end{array}$ \\
\hline
\end{tabular}

aDelayed recall was assessed using the five-word delayed recall component of the Montreal Cognitive Assessment, with probable impairment defined as the ability to recall two or fewer words after 5 minutes. ${ }^{5}$ Executive function was assessed using the Clock-in-the-Box test, with

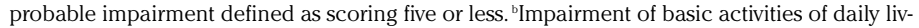
ing (ADL) and instrumental activities of daily living (IADL) was defined as patients reporting requiring assistance or being dependent on others to complete one or more of six ADL or five IADL, respectively.SD: standard deviation; ADL: basic activities of daily living; IADL: instrumental activities of daily living. 
for all patients could have contributed to a more effective geriatrics-led intervention.

Reducing mortality is not necessary to justify the integration of geriatrics into the care of patients with blood cancers. Emerging evidence from other trials of predominantly patients with solid tumors suggests that GA-driven interventions improve meaningful outcomes other than survival in older patients with cancer, including decreased treatment toxicity and improvements across multiple domains of quality of life..$^{16,17,27,28,36,37}$ Various models of GA-driven interventions were studied in these trials, ranging from a GA summary with recommended interventions carried out by the treating hematologic oncologist, to an embedded comanagement model led by a geriatrician (as described

A

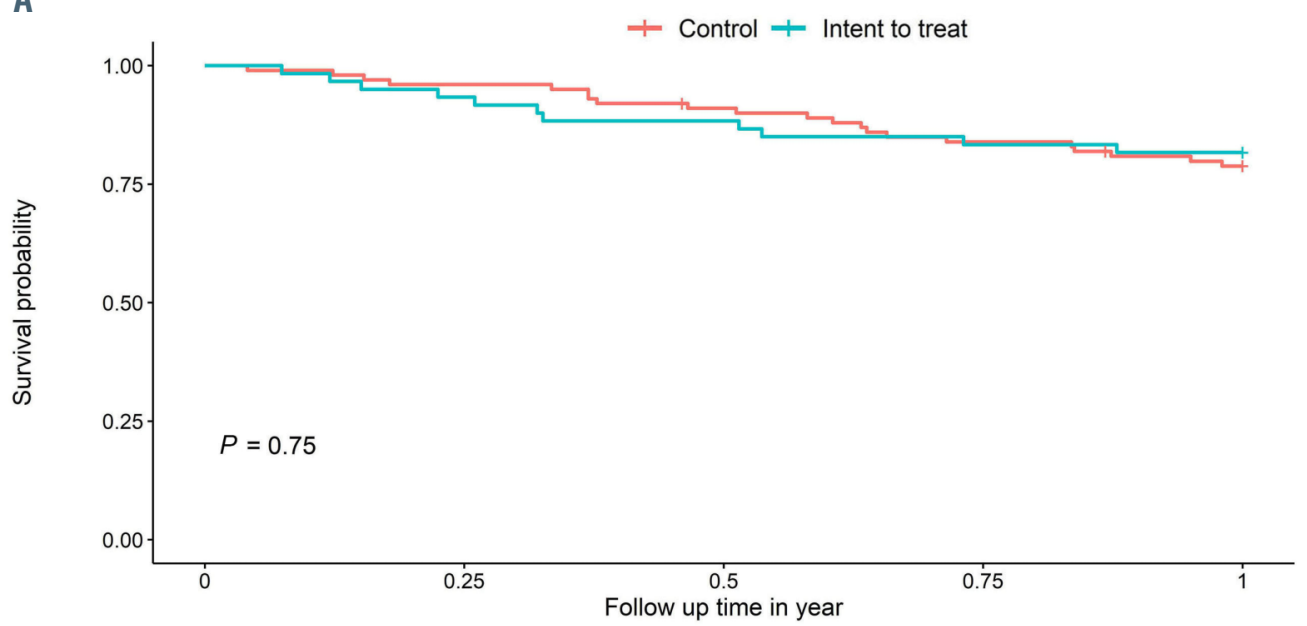

Intent to treat $\left[\begin{array}{ccccc}\text { Number at risk } \\ \qquad 0 & 96 & 90 & 83 & 77 \\ \hline 0 & 56 & 53 & 50 & 1\end{array}\right.$

B
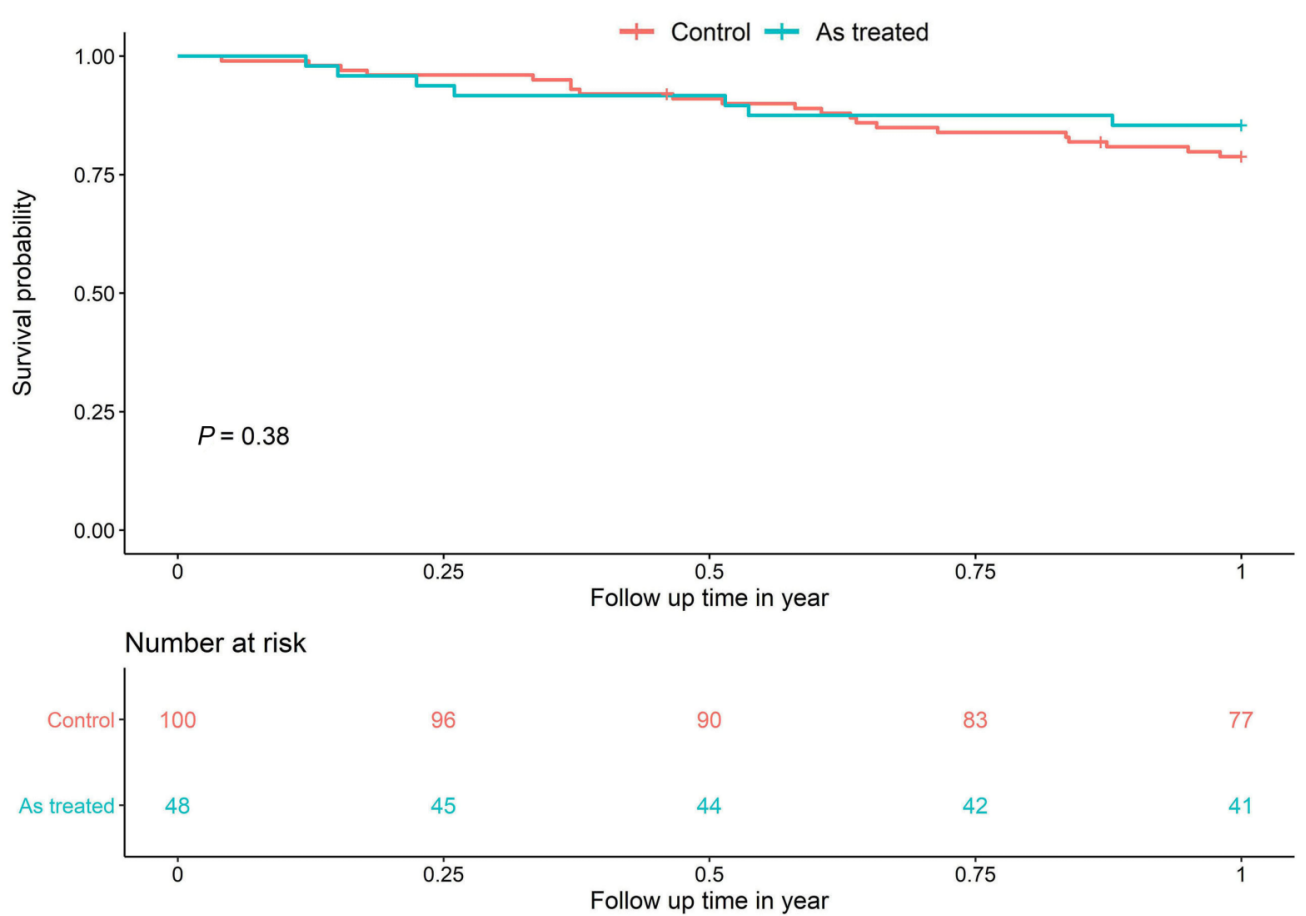

Figure 2. One-year overall survival by standard oncologic care (control) versus geriatric consultation + standard care. (A) Intent-to-treat analysis. (B) Per-protocol analysis. 
Table 2. Multivariable analyses assessing effect of geriatric consultation on overall mortality rate through 1 year of follow-up, acute care utilization, and goals-of-care discussions.

\begin{tabular}{|c|c|c|}
\hline $\begin{array}{l}\text { Overall mortality rate through } \\
1 \text { year }\end{array}$ & $\begin{array}{l}\text { Hazard ratio } \\
\text { (95\% CI) }\end{array}$ & P value \\
\hline Intent-to-treat $v s$. control & $0.93(0.45-1.95)$ & 0.85 \\
\hline Per-protocol vs control & $0.70(0.30-1.66)$ & 0.42 \\
\hline ED visits & $\begin{array}{c}\text { Incidence rate ratio } \\
\text { (95\% CI) }\end{array}$ & $P$ value \\
\hline Intent-to-treat $v s$. control & $0.89(0.33-2.42)$ & 0.82 \\
\hline Per-protocol vs. control & $0.77(0.26-2.23)$ & 0.62 \\
\hline Hospitalizations & $\begin{array}{c}\text { Incidence rate ratio } \\
(95 \% \text { CI) }\end{array}$ & P value \\
\hline Intent-to-treat $v s$. control & $0.91(0.30-2.71)$ & 0.86 \\
\hline Per-protocol us. control & $0.74(0.24-2.32)$ & 0.61 \\
\hline Days in hospital & $\begin{array}{c}\text { Incidence rate ratio } \\
\text { (95\% CI) }\end{array}$ & P value \\
\hline Intent-to-treat $v s$. control & $1.05(0.29-3.79)$ & 0.94 \\
\hline Per-protocol us. control & $0.82(0.20-3.38)$ & 0.78 \\
\hline EOL GOC discussions & Odds ratio (95\% CI) & P value \\
\hline Intent-to-treat $v s$. control & $3.12(1.03-9.41)$ & 0.05 \\
\hline Per-protocol vs. control & $3.58(1.13-11.35)$ & 0.03 \\
\hline
\end{tabular}

All models for the multivariable analyses were adjusted for age, sex, disease aggressiveness, and frailty. Separate models were run for per-protocol analysis. 95\% CI: 95\% confidence interval; ED: emergency department; EOL GOC: end-of-life goals of care.

above). Future trials in older adults with blood cancers should investigate not only the effectiveness of different models of geriatrics-driven interventions in terms of mortality, but also their impact on treatment decisions and patient-centered outcomes such as function and quality of life, which are outcomes often valued by older patients just as much as, if not more than, survival. ${ }^{38-41}$ Reducing toxicity and optimizing function and quality of life - all while maintaining similar survival in comparison with standard oncologic care - constitute a net benefit for complex older patients. ${ }^{42}$

To this end, our finding that geriatric consultation increased the likelihood of having documented EOL goalsof-care discussions is clinically relevant to pre-frail and frail older adults with blood cancers, many of whom have a high risk of death regardless of intervention. ${ }^{4,43}$ Discussing preferences regarding place of death and resuscitation status is of paramount importance in frail older patients with blood cancers; doing so early in the outpatient setting can reduce intensive care use in the days before death while increasing hospice enrollment. ${ }^{44}$ Moreover, a geriatrician's evaluation of age-related vulnerabilities (e.g., functional and cognitive impairment) and their potential reversibility better informs these goals-of-care discussions. ${ }^{42}$ Many frail older patients may have other advanced conditions that limit their prognosis independently of their cancer or its treatment, diminishing the benefits and increasing the harms of intensive chemotherapy. Indeed, our trial population had high rates of cognitive, functional, and mobility impairment, more representative of patients aged $\geq 75$ years treated in practice than the small number of patients in this age group enrolled in clinical trials. ${ }^{4,45}$

Beyond aligning EOL care with patients' preferences, the geriatricians' expertise in evaluation and management of age-related vulnerabilities was highly valued by surveyed hematologic oncologists and other clinicians at DanaFarber. Most rated geriatric consultation to be useful in the
Table 3. Survey results of oncologists' opinions regarding value of geriatric consultation. ${ }^{\text {a }}$

\begin{tabular}{lcc} 
Domains of care & $\begin{array}{c}\text { Number } \\
\text { of responses }\end{array}$ & $\begin{array}{c}\text { \% who answered } \\
\text { 4 or } 5 \text { (95\% Cl) }\end{array}$ \\
\hline Evaluating cognition & 35 & $85.7(69.7-95.2)$ \\
Connecting patients to resources & 35 & $80.0(63.1-91.6)$ \\
Diagnosing frailty & 35 & $77.1(59.9-89.6)$ \\
Managing non-oncologic comorbidities & 35 & $77.1(59.9-89.6)$ \\
Tailoring end-of-life care & 35 & $71.4(53.7-85.4)$ \\
Informing treatment decisions & 35 & $62.9(44.9-78.5)$ \\
Managgement of age-related issues & Number & $\%$ who answered \\
& of responses & 4 or $5(9.5 \%$ Cl) \\
Functional status & 35 & $88.2(72.6-96.7)$ \\
Falls & 35 & $85.7(69.7-95.2)$ \\
Depression & 35 & $80.0(63.1-91.6)$ \\
Mood disorders & 34 & $79.4(62.1-91.3)$ \\
Insomnia & 35 & $77.1(59.9-89.6)$ \\
Nutrition & 35 & $62.9(44.9-78.5)$ \\
Pain & 35 & $62.9(44.9-78.5)$ \\
\hline
\end{tabular}

${ }^{\mathrm{a}}$ For each question, responses were rated on a Likert scale ranging from $0=$ not at all useful to $5=$ very useful. CI: confidence interval.

evaluation of cognition, management of non-oncologic comorbidities, and management of functional status and falls. Fewer clinicians found geriatric consultation to be useful in informing oncologic treatment decisions and the management of nutrition and pain. The latter might in part be due to the comfort of hematologic oncology teams in treating these problems themselves, with support from nutritionists and other allied healthcare services.

Our study has limitations other than those related to the geriatric consultation model listed above. Our study took place at a large, academic, tertiary care center that may limit generalizability of its findings to community practices. However, GA-driven interventions have been shown to be feasible and improve outcomes in other settings, including community hematologic oncology clinics. ${ }^{14,28}$ Competing risk of mortality may have hindered observation of hospitalizations and other secondary outcomes; three patients in the consultation arm died before they could even receive the intervention. Although we did not detect a difference in care utilization between study arms, the study may have been underpowered to investigate these secondary outcomes. Indeed, our overall event rates for deaths and care utilization were low, likely because many of our patients were on observation for less aggressive disease. Future trials could further minimize heterogeneity in patients' characteristics by limiting enrollment to patients with one or two types of blood cancer on active treatment. Along with investigating patient-centered outcomes, future trials should also investigate the impact of GA-guided care on treatment toxicity, treatment discontinuation, and progression free survival.

In conclusion, our randomized trial of embedded geriatric consultation for pre-frail and frail older patients with blood cancers did not show an improvement in survival or healthcare utilization, but did increase EOL goals of care discussions and was valued by hematologic oncology clinicians. Lessons learned from our trial complemented by the results emerging from others suggest that ensuring earlier delivery and more longitudinal co-management may be necessary to have an impact on outcomes such as survival and hospitalizations. Such models should be investigated in older adults with blood cancers, along with their impact on patient-centered outcomes such as function and quality of life. Future 
studies should determine not only which models may be effective, but also whether certain components of GA-driven interventions are more effective than others. The benefits of different models must be balanced against their scalability, especially considering the current limitations in oncology practices' access to geriatricians. ${ }^{46,47}$ Such information will help oncology practices with varying resources adapt models of geriatric care that are feasible, effective, and sustainable in improving the care of older patients with blood cancers.

\section{Disclosures}

RJS serves on the Board of Directors for Kiadis and Be The Match/National Marrow Donor Program; has provided consulting for Gilead, Rheos Therapeutics, VOR Biopharma, and Takeda; and served on a Data Safety Monitoring Board for Juno/Celgene.

\section{Contributions}

$G A A, J A D, R M S$, and RJS designed the trial and oversaw execution of the protocol, contributed to data analysis and interpretation, and to preparing the manuscript; $C D$ contributed to data acquisition, analysis, and interpretation, and wrote manuscript; HU contributed to the trial design, data analysis and interpretation, and to preparing the manuscript; GZ analyzed data and contributed to interpretation and manuscript preparation; TH oversaw execution of the protocol, contributed to data acquisition and interpretation, and to preparing the manuscript;
$R C$ and $E M$ contributed to data acquisition and interpretation, and to preparing the manuscript; $L M$ and HJ oversaw execution of the protocol, and contributed to data interpretation and preparing the manuscript.

\section{Funding}

This work was supported by the Harvard Translational Research in Aging Training Program (National Institute on Aging of the National Institutes of Health: T32AG023480) (CD); the Dana-Farber/Harvard Cancer Center SPORE in Multiple Myeloma (National Cancer Institute of the National Institutes of Health: P50 CA100707) (CD); the Harvard Catalyst (GZ); the Harvard Clinical and Translational Science Center (National Center for Advancing Translational Sciences, National Institutes of Health Award UL 1TR002541) and financial contributions from Harvard University and its affiliated academic healthcare centers. The content is solely the responsibility of the authors and does not necessarily represent the official views of Harvard Catalyst, Harvard University and its affiliated academic healthcare centers, or the National Institutes of Health; the Older Adult Hematologic Malignancy Program is supported by the Murphy Family Fund from the Dana-Farber Cancer Institute (GAA)

\section{Data-sharing statement}

Data and protocol requests will be considered on a case by case basis and in accordance with the regulations of the Dana-Farber Harvard Cancer Center Office for Human Research Studies.

\section{References}

1. Surveillance, Epidemiology, and End Results (SEER) (2013-2017). Cancer Stat Facts: Myeloma. National Cancer Institute. Accessed June, 2020 https://seer.cancer.gov/statfacts/html/mul my.html

2. Surveillance, Epidemiology, and End Results (SEER) (2013-2017). Cancer Stat Facts: Leukemia. National Cancer Institute. Accessed June, 2020 https://seer.cancer.gov/statfacts/html/mul my.html

3. Surveillance, Epidemiology, and End Results (SEER) (2013-2017). Cancer Stat Facts: Non-Hodgkin Lymphoma. National Cancer Institute. Accessed June, 2020. https://seer.cancer.gov/statfacts/html/mul my.html

4. Abel GA, Klepin HD. Frailty and the management of hematologic malignancies. Blood. 2018;131(5):515-524.

5. Hshieh TT, Jung WF, Grande LJ, et al. Prevalence of cognitive impairment and association with survival among older patients with hematologic cancers. JAMA Oncol. 2018;4(5):686-693.

6. DuMontier C, Liu MA, Murillo A, et al. Function, survival, and care utilization among older adults with hematologic malignancies. J Am Geriatr Soc. 2019;67 (5):889-897.

7. Liu MA, DuMontier C, Murillo A, et al. Gait speed, grip strength, and clinical outcomes in older patients with hematologic malignancies. Blood. 2019;134(4):374-382.

8. Mohile SG, Dale W, Somerfield MR, et al. Practical assessment and management of vulnerabilities in older patients receiving chemotherapy: ASCO guideline for geriatric oncology. J Clin Oncol. 2018;36(22):2326-2347.

9. Repetto L, Fratino L, Audisio RA, et al.
Comprehensive geriatric assessment adds information to Eastern Cooperative Oncology Group performance status in elderly cancer patients: an Italian Group for Geriatric Oncology Study. J Clin Oncol. 2002;20(2):494-502

10. Hurria A, Mohile S, Gajra A, et al. Validation of a prediction tool for chemotherapy toxicity in older adults with cancer. J Clin Oncol. 2016;34(20):2366 2371.

11. Extermann M, Boler I, Reich RR, et al. Predicting the risk of chemotherapy toxicity in older patients: the Chemotherapy Risk Assessment Scale for High-Age Patients (CRASH) score. Cancer. 2012;118(13):3377-3386

12. Palumbo A, Bringhen S, Mateos MV, et al. Geriatric assessment predicts survival and toxicities in elderly myeloma patients: an International Myeloma Working Group report. Blood. 2015;125(13):2068-2074.

13. Mohile SG, Velarde C, Hurria A, et al. Geriatric assessment-guided care processes for older adults: a Delphi consensus of geriatric oncology experts. J Natl Compr Canc Netw 2015:13(9):1120-1130.

14. Mohile SG, Magnuson A, Pandya C, et al. Community oncologists' decision-making for treatment of older patients with cancer. J Natl Compr Canc Netw. 2018;16(3):301309.

15. Mohile SG, Epstein RM, Hurria A, et al Communication with older patients with cancer using geriatric assessment: a clusterrandomized clinical trial from the National Cancer Institute community oncology research program. JAMA Oncol. 2019;6(2): 196-204.

16. Corre R, Greillier L, Caër HL, et al. Use of a comprehensive geriatric assessment for the management of elderly patients with advanced non-small-cell lung cancer: the phase III randomized ESOGIA-GFPC-
GECP 08-02 study. J Clin Oncol. 2016;34 (13):1476-1483

17. Nipp RD, Temel B, Fuh C-X, et al. Pilot randomized trial of a transdisciplinary geriatric and palliative care intervention for older adults with cancer. J Natl Compr Canc Netw. 2020;18(5):591

18. Fried LP, Tangen CM, Walston J, et al. Frailty in older adults: evidence for a phenotype. J Gerontol A Biol Sci Med Sci. 2001:56(3):M146-156.

19. Rockwood K, Mitnitski A. Frailty in relation to the accumulation of deficits. Gerontol A Biol Sci Med Sci. 2007;62(7): 722-727.

20. Temel JS, Greer JA, Muzikansky A, et al. Early palliative care for patients with metastatic non-small-cell lung cancer. $\mathrm{N}$ Engl J Med. 2010;363(8):733-742.

21. Abel GA, Neufeld EJ, Sorel M, Weeks IC. Direct-to-consumer advertising for bleeding disorders: a content analysis and expert evaluation of advertising claims. J Thromb Haemost. 2008;6(10):1680-1684.

22. El-Jawahri AR, Abel GA, Steensma DP, et al. Health care utilization and end-of-life care for older patients with acute myeloid leukemia. Cancer. 2015;121(16):2840-2848.

23. Mack JW, Cronin A, Taback N, et al. Endof-life care discussions among patients with advanced cancer: a cohort study. Ann Int Med. 2012;156(3):204-210.

24. Uno H, Claggett B, Tian L, et al. Moving beyond the hazard ratio in quantifying the between-group difference in survival analysis. J Clin Oncol. 2014;32(22):2380-2385.

25. surv2sampleComp: Inference for ModelFree Between-Group Parameters for Censored Survival Data. R package version 1.0-5. 2017. https://CRAN.Rproject.org/package $=$ surv2sampleComp

26. Uno H, Cai T, Tian L, Wei LJ. Evaluating prediction rules for $\mathrm{t}$-year survivors with censored regression models. J Am Stat Ass. 
2007;102(478):527-537.

27. Li D, Sun C-L, Kim H, et al. Geriatric assessment-driven intervention (GAIN) on chemotherapy toxicity in older adults with cancer: A randomized controlled trial. J Clin Oncol. 2020;38(15_suppl):12010.

28. Mohile SG, Mohamed MR, Culakova E, et al. A geriatric assessment (GA) intervention to reduce treatment toxicity in older patients with advanced cancer: a University of Rochester Cancer Center NCI community oncology research program cluster randomized clinical trial (CRCT). J Clin Oncol. 2020;38(15_suppl):12009.

29. Soo WK, King M, Pope A, et al. The Elderly Functional Index (ELFI), a patient-reported outcome measure of functional status in patients with cancer: a multicentre, prospective validation study. Lancet Healthy Longev. 2021;2(1):e24-e33.

30. Shahrokni A, Tin AL, Sarraf S, et al. Association of geriatric comanagement and 90-day postoperative mortality among patients aged 75 years and older with cancer. JAMA Netw Open. 2020;3(8):e209265.

31. Lund CM, Vistisen KK, Olsen AP, et al. The effect of geriatric intervention in frail older patients receiving chemotherapy for colorectal cancer: a randomised trial (GERICO). Br J Cancer. 2021;124(12):1949-1958.

32. Derman BA, Kordas K, Ridgeway J, et al. Results from a multidisciplinary clinic guided by geriatric assessment before stem cell transplantation in older adults. Blood Adv. 2019;3(22):3488-3498.

33. Clarfield AM, Bergman H, Kane R.
Fragmentation of care for frail older people-an international problem. Experience from three countries: Israel, Canada, and the United States. J Am Geriatr Soc. 2001;49 (12):1714-1721

34. Dale W, Chow S, Sajid S. Socioeconomic considerations and shared-care models of cancer care for older adults. Clin Geriatr Med. 2016;32(1):35-44.

35. Presley CJ, Krok-Schoen JL, Wall SA, et al. Implementing a multidisciplinary approach for older adults with cancer: geriatric oncology in practice. BMC Geriatr. 2020;20(1):231.

36. Soo W-K, King M, Pope A, Parente P, Darzins P, Davis ID. Integrated geriatric assessment and treatment (INTEGERATE) in older people with cancer planned for systemic anticancer therapy. J Clin Oncol. 2020;38(15_suppl):12011.

37. Qian CL, Knight HP, Ferrone CR, et al. Randomized trial of a perioperative geriatric intervention for older adults with cancer. J Clin Oncol. 2020;38(15_suppl):12012.

38. Fried TR, Bradley EH, Towle VR, Allore H. Understanding the treatment preferences of seriously ill patients. N Engl J Med. 2002;346(14):1061-1066.

39. Loh KP, Mohile SG, Epstein RM, et al Willingness to bear adversity and beliefs about the curability of advanced cancer in older adults. Cancer. 2019;125(14):25062513.

40. Celis ESPD, Li D, Sun C-L, et al. Patientdefined goals and preferences among older adults with cancer starting chemotherapy
(CT). J Clin Oncol. 2018;36(15_suppl): 10009.

41. Rosko AE, Wall S, Baiocchi R, et al. Aging phenotypes and restoring functional deficits in older adults with hematologic malignancy. J Natl Compr Canc Netw. 2021;19(9):1027-1036.

42. DuMontier C, Loh KP, Bain PA, et al Defining undertreatment and overtreatment in older adults with cancer: a scoping literature review. J CLin Oncol. 2020;38(22):2558-2569.

43. Krok-Schoen JL, Fisher JL, Stephens JA, et al. Incidence and survival of hematological cancers among adults ages $>/=75$ years Cancer Med. 2018;7(7):3425-3433.

44. Odejide OO, Uno H, Murillo A, Tulsky JA, Abel GA. Goals of care discussions for patients with blood cancers: association of person, place, and time with end-of-life care utilization. Cancer. 2020;126(3):515-522.

45. Ludmir EB, Mainwaring W, Lin TA, et al Factors associated with age disparities among cancer clinical trial participants. JAMA Oncol. 2019;5(12):1769-1773.

46. Williams GR, Weaver KE, Lesser GJ, et al. Capacity to provide geriatric specialty Care for Older Adults in Community Oncology Practices. Oncologist. 2020;25(12):10321038.

47. Dale W, Williams GR, MacKenzie AR, et al. How is geriatric assessment used in clinical practice for older adults with cancer? A survey of cancer providers by the American Society of Clinical Oncology. JCO Oncol Pract. 2021;17(6):336-344. 